\title{
POTENTIALS AND THE RANDOM WALK
}

\author{
BY \\ Kıyosi Itô AND H. P. MCKeAN, JR. ${ }^{1}$
}

\section{Introduction}

Given an integer $s \geqq 3$, write $e_{1}, e_{2}, \cdots, e_{s}$ for the $s$ coordinate vectors $(1,0, \cdots, 0),(0,1, \cdots, 0), \cdots,(0,0, \cdots, 1)$, spanning the $s$-dimensional lattice of points with integral coordinates, and let $s_{n}$ denote the position at time $n(=0,1,2, \cdots)$ of a particle performing the standard $s$-dimensional random walk according to the following rule: fixing the first $n-1$ steps $s_{1}, s_{2}, \cdots, s_{n-1}$, the particle starts afresh at $s_{n-1}$, jumping next to one of the $2 s$ neighbors $s_{n}=s_{n-1} \pm e_{1}, s_{n-1} \pm e_{2}, \cdots, s_{n-1} \pm e_{s}$ of $s_{n-1}$, the chance of landing at a particular neighbor being $(2 s)^{-1}$.

Given a set $B$ of lattice points, the probability $p_{B}$ that the random walk hits $B$ at some time $n<+\infty$, as a function of the starting point of the walk, is excessive in the sense that $\mathbf{G} p_{B} \leqq 0$, where $\mathbf{G}$ is Laplace's difference operator:

$$
(\mathbf{G} p)(a)=(2 s)^{-1} \sum_{k \leqq s, n=1,2} p\left(a+(-)^{n} e_{k}\right)-p(a) .
$$

B. H. Murdoch [1, pp. 13-19] proved that if $p \geqq 0$, and if $\mathbf{G} p=0$, then $p$ is constant, ${ }^{2}$ and, with the help of this result, it follows, as Murdoch himself noted, that $p_{B}$ is the sum of the potential $\mathbf{K} e_{B}$ and the constant $p_{B}(\infty)$, where $e_{B}=-\mathbf{G} p_{B}(\geqq 0), \mathbf{K} e_{B}$ is the expectation of $\sum_{n \geqq 0} e_{B}\left(s_{n}\right)$, as a function of the starting point of the walk, and $p_{B}(\infty)$ is the (constant) probability $P$.(B) of the event $\mathbf{B}$ that $s_{n} \in B$ for an infinite number of integers $n$.

$P$.(B) is either 0 or 1 . When $P .(\mathbf{B})=0, p_{B}$ is the greatest potential $p \leqq 1$ such that $\mathbf{G} p=0$ outside $B$, and, on the strength of the example of the Newtonian potential in 3 dimensions, it is natural to think of $e_{B}$ as the electrostatic distribution of charge on the conductor $B$ and to introduce the total charge (of $e_{B}$ ) as the capacity $C(B)$ of $B$.

Given a set $B$, it is an interesting problem to decide whether $P .(\mathbf{B})=0$ or 1 ; the solution is

$$
1.2 P \text {.(B) }=0 \text { or } 1 \text { according as } \sum_{n \geqq 0} 2^{-n(s-2)} C\left(B_{n}\right)<\text { or }=+\infty \text {, }
$$

where $B_{n}$ is the intersection of $B$ and the spherical shell $2^{n} \leqq|a|<2^{n+1}$. Wiener's test for the singular points of the Newtonian electrostatic potential (see Courant and Hilbert [1, p. 286]) served us as a model, and for this reason we call 1.2 Wiener's test also. B. H. Murdoch [1, pp. 45-47] came close to proving 1.2 and used his method to compute $P$.(B) for sets $B$ similar to those

Received September 25, 1958.

${ }^{1}$ Fulbright grantee 1957-1958.

${ }^{2} \mathrm{~J}$. Capoulade [1] also stated this result and S. Verblunsky [1] and R. Duffin [1, pp. 242-245] proved it. Murdoch's results lie much deeper. 
figuring in the last example of Section 6. Similar results hold for the Brownian motion and Newtonian potentials in $s(\geqq 2)$ dimensions and for stable processes with exponent $<2(<1)$ and Riesz potentials in $s \geqq 2(\geqq 1)$ dimensions; for the identification of hitting probabilities and electrostatic potentials, see J. L. Doob [2] and G. Hunt [1, 2]; the proof of Wiener's test runs along the lines of Section 5. G. Hunt $[1,2,3]$ showed the full scope and power of the connection between Markov processes and potentials, and most of the (nonclassical) results of Sections 3 and 4 are from his papers [1,2] or from B. H. Murdoch. The present paper is based on lectures given at Kyôto and Fukuoka in April, 1958, in which we tried to present Hunt's things in the simplest setting.

We thank J. L. Doob, N. Ikeda, and D. Ray for spotting several misprints.

\section{The random walk}

Write $W$ for the space of paths $w: n=1,2, \cdots \rightarrow s_{n}(w)$ with values from the $s$-dimensional lattice, $w_{m}^{-}$for the stopped path $s_{n}\left(w_{m}^{-}\right)=s_{n \wedge m}(w){ }^{3}$ $w_{m}^{+}$for the shifted path $s_{n}\left(w_{m}^{+}\right)=s_{n+m}(w)$, C for the class of Borel subsets of $W$, and, for $\mathbf{C}$ measurable $m=m(w) \geqq 0$, write $\mathbf{C}_{m}$ for the Borel algebra of sets

$$
A=\left(w: \overline{w_{m}} \epsilon C\right),
$$

let $P .(C)$ denote the probability of the event $C \in \mathbf{C}$ for the standard random walk as a function of the starting point of the walk; note that $P .\left(w_{n}^{+} \epsilon C / \mathrm{C}_{n}\right)=$ $P_{s_{n}}(C)$ for each $n \geqq 0$ and each $C \in \mathbf{C}$; and let $E .(f)=\int f P .(d w)$.

$m=m(w)=0,1,2, \cdots$ is a Markov time if $(w: m>n) \epsilon \mathbf{C}_{n}$ for each $n \geqq 0$; for example, given a set $B$ of lattice points, $m=n_{B}=\min \left(n: s_{n} \in B\right)$ is Markov.

Given a Markov time $m$,

$$
P .\left(P .\left(w_{m}^{+} \epsilon C / \mathbf{C}_{m}\right)=P_{s_{m}}(C)\right)=1,
$$

in short, $s_{n}: n \geqq 0$ starts from scratch at time $n=m$ at the place $s_{m}$.

The proof is simple: $(w: m=n) \epsilon \mathbf{C}_{n}$ for each $n \geqq 0$, and therefore

$$
\begin{aligned}
P .\left(w_{m}^{+} \epsilon C^{+}, w_{m}^{-} \epsilon C^{-}\right) & =\sum_{n \geqq 0} P .\left(w_{n}^{+} \epsilon C^{+}, w_{n}^{-} \epsilon C^{-}, m=n\right) \\
& =\sum_{n \geqq 0} E .\left(P_{s_{n}}\left(C^{+}\right), w_{n}^{-} \epsilon C^{-}, m=n\right) \\
& =E .\left(P_{s_{m}}\left(C^{+}\right), w_{m}^{-} \epsilon C^{-}\right), \quad C^{-}, C^{+} \epsilon \mathbf{C} .
\end{aligned}
$$

Given a point $\theta=\left(\theta_{1}, \theta_{2}, \cdots, \theta_{s}\right)$ of the $s$-dimensional torus $[-\pi, \pi)^{s}$,

$$
\begin{array}{r}
E_{a}\left(e^{i s_{n} \cdot \theta}\right)=E_{a}\left(E_{s_{n-1}}\left(e^{i s_{1} \cdot \theta}\right)\right)=E_{a}\left(e^{i s_{n-1} \cdot \theta}\right) f(\theta)=e^{i a \cdot \theta} f(\theta)^{n}, \\
f(\theta)=s^{-1} \sum_{k \leqq s} \cos \theta_{k}, \quad n \geqq 0 .{ }^{4}
\end{array}
$$

\footnotetext{
${ }^{3} m \wedge n$ means the smaller of $m$ and $n$.

${ }^{4} s_{n} \cdot \theta$ is the inner product of the vectors $s_{n}$ and $\theta$.
} 
and inverting 2.3 proves the result of G. Pólya [1, pp. 151-153]:

$$
P_{a}\left(s_{n}=b\right)=(2 \pi)^{-s} \int e^{i(b-a) \cdot \theta} f(\theta)^{n} d \theta, \quad n \geqq 0 .
$$

Summing 2.4 for $n=0,1,2, \cdots$ proves

$$
\begin{aligned}
K(a, b)=\sum_{n \geqq 0} P_{a}\left(s_{n}=b\right) & =(2 \pi)^{-s} \int e^{i(b-a) \cdot \theta} \frac{d \theta}{g(\theta)} \\
\leqq & (2 \pi)^{-s} \int \frac{d \theta}{g(\theta)}<+\infty, \quad g(\theta)=1-f(\theta),
\end{aligned}
$$

and using the Borel-Cantelli lemma, we infer that

$$
\text { P. }\left(\lim _{n \uparrow+\infty} s_{n}=\infty\right)=1 .
$$

B. H. Murdoch [1, pp. 23-32] and (for $s=3$ ) R. Duffin [1, pp. 238-240, 245-251] estimated $K(a, b)$ for $|a-b| \uparrow+\infty$ up to terms of magnitude $|a-b|^{-s-2}$.

We will want the following simpler result:

$$
\begin{aligned}
\lim _{|b-a| \uparrow+\infty}|b-a|^{s-2} K(a, b) & |b-a|^{s-2}(2 \pi)^{-s} \int e^{i(b-a) \cdot \theta} \frac{d \theta}{g(\theta)}=k_{1}, \\
= & \lim _{|b-a| \uparrow+\infty} \mid b-\int_{1}=s \int_{0}^{+\infty}(2 \pi t)^{-s / 2} e^{-1 / 2 t} d t^{5}
\end{aligned}
$$

Consider, for the proof, the modified Bessel coefficients

2.8

$$
I_{n}(t)=\frac{1}{2 \pi} \int_{-\pi}^{\pi} e^{i n \theta} e^{t \cos \theta} d \theta
$$

$$
=\frac{e^{t}}{2 \pi} \int_{-\pi}^{\pi} e^{i n \theta} e^{-2 t \sin ^{2}(\theta / 2)} d \theta, \quad n=0, \pm 1, \pm 2, \cdots,
$$

introduce the (positive) Fourier coefficients

$$
\begin{aligned}
& s(2 \pi)^{-s} \int e^{i c \cdot \theta} e^{-s t g(\theta)} d \theta \\
& \quad=s(2 \pi)^{-1} \int_{-\pi}^{\pi} e^{i l_{1} \theta_{1}} e^{-2 t \sin ^{2}\left(\theta_{1} / 2\right)} d \theta_{1} \cdots(2 \pi)^{-1} \int_{-\pi}^{\pi} e^{i l_{s} \theta_{s}} e^{-2 t \sin ^{2}\left(\theta_{s} / 2\right)} d \theta_{s} \\
& =s e^{-t} I_{l_{1}}(t) e^{-t} I_{l_{2}}(t) \cdots e^{-t} I_{l_{s}}(t), \quad t \geqq 0, \quad c=\left(l_{1}, l_{2}, \cdots, l_{s}\right),
\end{aligned}
$$

and note that

$$
(2 \pi)^{-s} \int e^{i c \cdot \theta} \frac{d \theta}{g(\theta)}=\int_{0}^{+\infty} d t s(2 \pi)^{-s} \int e^{i c \cdot \theta} e^{-s \operatorname{tg}(\theta)} d \theta
$$

$$
=s \int_{0}^{+\infty} e^{-s t} d t I_{l_{1}}(t) I_{l_{2}}(t) \cdots I_{l_{s}}(t) .
$$

${ }^{5} k_{1}, k_{2}, \cdots$ denote positive constants. 
Given $\theta \epsilon[-\pi, \pi)^{s}, g(\theta)>k_{2}|\theta|^{2}$, so that

$$
e^{-s t|c|^{2} g(\theta /|c|)}<e^{-s t k_{2}|\theta|^{2}}, \quad t \geqq 0, \quad \theta \in[-\pi|c|, \pi|c|)^{s},
$$

and

$$
\begin{aligned}
\left.|| c\right|^{s-2} \int_{k_{3}|c|^{2}}^{+\infty} s(2 \pi)^{-s} \int_{[-\pi, \pi)^{s}} e^{i c \cdot \theta} e^{-s t g(\theta)} d \theta d t & \\
& \quad-\int_{k_{3}}^{+\infty} s(2 \pi)^{-s} \int_{|\theta| \leqq \pi|c|} e^{i(c /|c|) \cdot \theta} e^{-(t / 2)|\theta|^{2}} d \theta d t \mid \\
\leqq & s(2 \pi)^{-s} \int_{k_{3}}^{+\infty} d t \mid \int_{[-\pi|c|, \pi|c|)^{s}} e^{i(c /|c|) \cdot \theta} e^{-s t|c|^{2} g(\theta /|c|)} d \theta
\end{aligned}
$$

2.12

$$
-\int_{|\theta| \leqq \pi|c|} e^{i(c /|c|) \cdot \theta} e^{-(t / 2)|\theta|^{2}} d \theta \mid
$$

$$
\begin{aligned}
\leqq s(2 \pi)^{-s} \int_{k_{3}}^{+\infty} d t\left[\int_{|\theta| \leqq \pi|c|}\left|e^{-s t|c|^{2} g(\theta|| c \mid)}-e^{-(t / 2)|\theta|^{2}}\right| d \theta\right. \\
\left.+\int_{\pi|c|<|\theta|<s^{1 / 2} \pi|c|} e^{-s t k_{2}|\theta|^{2}} d \theta\right]
\end{aligned}
$$

$\rightarrow 0$,

$$
|c| \uparrow+\infty
$$

this implies

$$
\begin{aligned}
\lim _{|c| \uparrow+\infty}|c|^{s-2} \int_{k_{3}|c|^{2}}^{+\infty} s(2 \pi)^{-s} \int_{[-\pi, \pi)^{s}} e^{i c \cdot \theta} e^{-s t t_{g}(\theta)} d \theta d t \\
=\int_{k_{3}}^{+\infty} d t s(2 \pi)^{-s} \int_{R^{s}} e^{i \theta_{1}} e^{-(t / 2)|\theta|^{2}} d \theta \\
=\int_{k_{3}}^{+\infty} s(2 \pi t)^{-s / 2} e^{-1 / 2 t} d t \\
\uparrow s \int_{0}^{+\infty}(2 \pi t)^{-s / 2} e^{-1 / 2 t} d t=k_{1},
\end{aligned}
$$

and to complete the proof, it is enough to check that

$2.14 \lim _{k_{3} \downarrow 0} \limsup _{|c| \uparrow+\infty}|c|^{s-2} \int_{0}^{k_{3}|c|^{2}} d t s(2 \pi)^{-s} \int_{[-\pi, \pi)^{s}} e^{i c \cdot \theta} e^{-s t_{g}(\theta)} d \theta=0$.

But, as is clear from 2.8 and 2.9 ,

$$
\begin{array}{rl}
\limsup _{|c| \uparrow+\infty}|c|^{s-2} \int_{0}^{k_{3}|c|^{2}} & d t s(2 \pi)^{-s} \int_{[-\pi, \pi) s} e^{i c \cdot \theta} e^{-s t g(\theta)} d \theta \\
& \leqq s^{s-1} \lim _{n \uparrow+\infty} n^{s-2} \int_{0}^{k_{3} s^{2} n^{2}} e^{-t} I_{n}(t)\left(e^{-t} I_{0}(t)\right)^{s-1} d t
\end{array}
$$

where $n$ is the greatest of the integers $\left|l_{1}\right|,\left|l_{2}\right|, \cdots,\left|l_{s}\right|$, and since, in view of 2.13 , 


$$
\lim _{n \uparrow+\infty} n^{s-2} s \int_{k_{3} s^{2} n^{2}}^{+\infty} e^{-t} I_{n}(t)\left(e^{-t} I_{0}(t)\right)^{s-1} d t
$$

$$
=\lim _{n \uparrow+\infty} n^{s-2} s \int_{k_{3} s^{2} n^{2}}^{+\infty}(2 \pi)^{-s} \int_{[-\pi, \pi)^{s}} e^{i n \theta_{1}} e^{-s t g(\theta)} d \theta d t
$$

$\uparrow k_{1}$,

it is enough to show that

$$
k_{1} \geqq \limsup _{n \uparrow+\infty} n^{s-2} s \int_{0}^{+\infty} e^{-t} I_{n}(t)\left(e^{-t} I_{0}(t)\right)^{s-1} d t .
$$

With the help of

$$
1 \geqq e^{-t} I_{0}(t)=\frac{1}{2 \pi} \int_{0}^{2 \pi} e^{-2 t \sin ^{2}(\theta / 2)} d \theta \sim(2 \pi t)^{-1 / 2}, \quad t \uparrow+\infty,
$$

and of

$$
\begin{aligned}
& \int_{0}^{r} e^{-t} I_{n}(t) d t=\frac{1}{2 \pi} \int_{0}^{2 \pi} e^{i n \theta} \frac{1-e^{-2 r \sin ^{2}(\theta / 2)}}{2 \sin ^{2}(\theta / 2)} d \theta \leqq n^{-s}, \\
& n \uparrow+\infty, \quad+\infty>r \geqq 0,
\end{aligned}
$$

our task now simplifies to showing that

$$
k_{1} \geqq \lim _{n \uparrow+\infty} \sup _{n} n^{s-2} s \int_{0}^{+\infty} e^{-t} I_{n}(t)(2 \pi t)^{-(s-1) / 2} d t,
$$

and, consulting A. Erdélyi [2, p. 196 (8) and 1, p. 164 (20)], it is seen that

$$
s \int_{0}^{+\infty} e^{-t} I_{n}(t)(2 \pi t)^{-(s-1) / 2} d t \sim k_{1} n^{2-s}, \quad n \uparrow+\infty,
$$

which completes the proof.

\section{Potentials}

Write $e$ for nonnegative functions defined for the points of the $s$-dimensional lattice, and consider the Green operator $\mathbf{K}$ defined in

$$
(\mathbf{K} e)(a)=E_{a}\left(\sum_{n \geqq 0} e\left(s_{n}\right)\right)
$$

$$
=\sum\left[\sum_{n \geqq 0} P_{a}\left(s_{n}=b\right)\right] e(b)=\sum K(a, b) e(b) .
$$

$-\mathbf{G}$ is inverse to $\mathbf{K}$; for, if $\mathbf{K} e<+\infty$, then

$$
\begin{aligned}
-\mathbf{G K} e & =E .\left(\sum_{n \geqq 0} e\left(s_{n}\right)\right)-E . E_{s_{1}}\left(\sum_{n \geqq 0} e\left(s_{n}\right)\right) \\
& =E .\left(\sum_{n \geqq 0} e\left(s_{n}\right)\right)-E .\left(\sum_{n \geqq 0} e\left(s_{n+1}\right)\right) \\
& =E .\left(e\left(s_{0}\right)\right)=e .
\end{aligned}
$$

A nonnegative function $p$ is excessive if $\mathbf{G} p \leqq 0$; it is a potential if $p=\mathbf{K} e$ with $e \geqq 0$; if $p=\mathbf{K} e(e \geqq 0, p<+\infty)$, then, as the reader will check, $\mathbf{G} p=-e \leqq 0$, so that potentials are excessive. 
Given an excessive function $p$,

$$
\begin{aligned}
p & =E \cdot\left(\sum_{l \leqq n}\left(p\left(s_{l-1}\right)-p\left(s_{l}\right)\right)+p\left(s_{n}\right)\right) \\
& =\sum_{l \leqq n} E \cdot\left(p\left(s_{l-1}\right)-E_{s_{l-1}}\left(p\left(s_{1}\right)\right)\right)+E \cdot\left(p\left(s_{n}\right)\right) \\
& =E \cdot\left(\sum_{l \leqq n}-(\mathbf{G} p)\left(s_{l-1}\right)\right)+E \cdot\left(p\left(s_{n}\right)\right),
\end{aligned}
$$

and, after putting $-\mathbf{G} p=e$ and $p_{\infty}=\lim _{n \uparrow+\infty} E .\left(p\left(s_{n}\right)\right)$, it results that

$$
p=\mathrm{K} e+p_{\infty} \text {. }
$$

$p_{\infty}$ is constant; indeed, it is nonnegative, $\mathbf{G} p_{\infty}=0$, and, as B. H. Murdoch proved, such a nonnegative harmonic function is constant.

J. L. Kelley's proof ${ }^{6}$ that a compact convex set is the convex hull of its extreme points served us as a model for the following simple proof of Murdoch's result.

Write $C$ for the class of nonnegative $p$ with $p(0)=1$ and $\mathbf{G} p=0$, label the points $c=\left(l_{1}, l_{2}, \cdots, l_{s}\right)$ of the $s$-dimensional lattice $c_{1}(=0), c_{2}, c_{3}, \cdots$, and, using the compactness that the estimate

$$
p\left(l_{1}, l_{2}, \cdots, l_{s}\right) \leqq(2 s)^{\left|l_{1}\right|+\left|l_{2}\right|+\ldots+\left|l_{s}\right|}, \quad p \in C,
$$

provides, put $m_{1}=1, C_{1}=C, m_{2}=\max _{p \epsilon C_{1}} p\left(c_{2}\right), C_{2}=C_{1} \mathbf{n}\left(p: p\left(c_{2}\right)=m_{2}\right)$, $m_{3}=\max _{p \epsilon C_{2}} p\left(c_{3}\right), C_{3}=C_{2} \cap\left(p: p\left(c_{3}\right)=m_{3}\right)$, etc., and select $p_{*} \in \bigcap_{n \geqq 1} C_{n}$. $p_{*}\left( \pm e_{k}\right)$ is then $>0, p_{*}\left( \pm e_{k}\right)^{-1} p_{*}\left(\cdot \pm e_{k}\right) \in C$ for each $k \leqq s$, and

$$
p_{*}=\sum_{k \leqq s, n=1,2}(2 s)^{-1} p_{*}\left((-)^{n} e_{k}\right) \frac{p_{*}\left(\cdot+(-)^{n} e_{k}\right)}{p_{*}\left((-)^{n} e_{k}\right)} .
$$

Since $\sum_{k \leqq s, n=1,2}(2 s)^{-1} p_{*}\left((-)^{n} e_{k}\right)=p_{*}(0)=1$, the definition of $p_{*}$ now implies that $p_{*}\left( \pm e_{k}\right)^{-1} p_{*}\left(\cdot \pm e_{k}\right) \epsilon \bigcap_{n \geqq 1} C_{n}$ for each $k \leqq s$; in short, $p_{*}\left( \pm e_{k}\right)^{-1} p_{*}\left(\cdot \pm e_{k}\right)=p_{*}$ for each $k \leqq s$, and we infer that

$$
p_{*}\left(l_{1}, l_{2}, \cdots, l_{s}\right)=p_{*}\left(e_{1}\right)^{l_{1}} p_{*}\left(e_{2}\right)^{l_{2}} \cdots p_{*}\left(e_{s}\right)^{l_{s}} \text {. }
$$

But

$3.61=p_{*}(0)=E_{0}\left(p_{*}\left(s_{1}\right)\right)=(1 / s) \sum_{k \leqq s} \frac{1}{2}\left(p_{*}\left(e_{k}\right)^{-1}+p_{*}\left(e_{k}\right)\right)$,

proving that $p_{*}\left(e_{k}\right)=1$ for each $k \leqq s$; therefore $p_{*} \equiv 1$; since $c_{2}$ was chosen at pleasure, each $p \in C$ is $\leqq 1$; and since, for $p \epsilon C, \mathbf{G} p \equiv 0, p \equiv 1$ is the sole member of $C$.

Keeping this result in mind, it is clear from 3.2 that, with the notation $p(\infty)=\lim _{\inf _{c \rightarrow \infty}} p(c)$,

$3.7 p(\infty) \geqq p_{\infty}=\lim _{n \uparrow+\infty} E \cdot\left(p\left(s_{n}\right)\right) \geqq E \cdot\left(\lim _{\inf _{n \uparrow+\infty}} p\left(s_{n}\right)\right) \geqq p(\infty)$, and 3.2 goes over into

$$
p=\mathbf{K} e+p(\infty), \quad p(\infty)=\lim _{n \uparrow+\infty} E .\left(p\left(s_{n}\right)\right) .
$$

${ }^{6}$ See P. T. Bateman [1, pp. 14-15]. 
Given a set $B$ of lattice points, let $n_{B}$ denote the hitting time min $\left(n: s_{n} \epsilon B\right)$ and $p_{B}$ the hitting probability $P .\left(n_{B}<+\infty\right)$.

$p_{B}$ is excessive; in fact,

$$
\begin{aligned}
e_{B} & =-\mathbf{G} p_{B}=p_{B}-E \cdot\left(p_{B}\left(s_{1}\right)\right) \\
& =P \cdot\left(n_{B}<+\infty\right)-P \cdot\left(n_{B}\left(w_{1}^{+}\right)<+\infty\right) \\
& =P .\left(n_{B}<+\infty, n_{B}\left(w_{1}^{+}\right)=+\infty\right) \geqq 0,
\end{aligned}
$$

and it follows from 2.6 and 3.9 that $e_{B}=0$ off the points of ${ }^{7} \partial B$ neighboring the (connected) part of the complement of $B$ reaching out to $\infty$.

Writing $\mathbf{B}$ for the event, $\bigcap_{n \geqq 1}\left(w: n_{B}\left(w_{n}^{+}\right)<+\infty\right)$ that $s_{n} \in B$ for an infinite number of times $n$, it results from

$3.10 p_{B}(\infty)=\lim _{n \uparrow+\infty} E .\left(p_{B}\left(s_{n}\right)\right)=\lim _{n \uparrow+\infty} P .\left(n_{B}\left(w_{n}^{+}\right)<+\infty\right)=P .(\mathbf{B})$

and from

$$
\begin{aligned}
P .(\mathbf{B}) & =\lim _{n \uparrow+\infty} P .\left(n_{B}<+\infty, n_{B}\left(w_{n_{B}+n}^{+}\right)<+\infty\right) \\
& =\lim _{n \uparrow+\infty} E .\left(n_{B}<+\infty, P_{s_{n_{B}}}\left(n_{B}\left(w_{n}^{+}\right)<+\infty\right)\right) \\
& =p_{B} P . \text { (B) }
\end{aligned}
$$

that $p_{B}(\infty)=P$.(B) is 0 or $1 . .^{8}$

Using these results, it is not difficult to prove that, for excessive $p=\mathbf{K} e+p(\infty)$,

$$
P \cdot\left(\lim _{n \uparrow+\infty} p\left(s_{n}\right)=p(\infty)\right)=1 ;^{9}
$$

indeed, $p \geqq p(\infty)$, and if $\alpha>p(\infty)$, if $A$ is the set where $p \geqq \alpha$, and if $p_{A}(\infty)=P .(\mathbf{A})=1$, then

$$
\left(w: l \leqq n \wedge n_{A}\right) \epsilon \mathbf{C}_{l},
$$

and

3.14

$$
\begin{aligned}
p= & E \cdot\left[\sum_{l \leqq n \wedge n_{A}}\left(p\left(s_{l-1}\right)-p\left(s_{l}\right)\right)+p\left(s_{n_{\wedge} n_{A}}\right)\right] \\
= & E \cdot\left[\sum_{l \leqq n \wedge n_{A}} e\left(s_{l-1}\right)\right]+E \cdot\left(p\left(s_{n_{A}}\right), n_{A} \leqq n\right) \\
& +E \cdot\left(p\left(s_{n}\right), n<n_{A}\right) \\
\geqq & \alpha P \cdot\left(n_{A} \leqq n\right) \uparrow \alpha p_{A}=\alpha, \quad n \uparrow+\infty,
\end{aligned}
$$

violating $\alpha>p(\infty)$, and we infer that $P$.(A) $=0$ for each $\alpha>p(\infty)$, completing the proof.

We give the proof of the general maximum principle of which 3.14 is a special case.

${ }^{7} \partial B$ is the set of points of $B$ not all of whose neighbors belong to $B$.

${ }^{8} P$.(B) $=0$ or 1 is a special case of the 0 -or- 1 law of Hewitt and Savage [1, pp. 493494].

${ }^{9} 3.12$ is a special case of the result of J. L. Doob [1, pp. 324-326] that a nonnegative lower semimartingale converges. 
Given excessive $p_{1}=\mathbf{K} e_{1}+p_{1}(\infty), p_{2}=\mathbf{K} e_{2}+p_{2}(\infty)$, if $p_{2} \geqq p_{1}$ on the support $B$ of $e_{1}$, and if $p_{2}(\infty) \geqq p_{1}(\infty)$ in case $P$.(B) $=0$, then $p_{2} \geqq p_{1}$ on the whole of the $s$-dimensional lattice; in fact, $e_{1}\left(s_{n}\right)=0$ for $n<n_{B}$, and by using the fact that, for excessive $p, p \geqq p(\infty)$ and $\lim _{n \uparrow+\infty} E \cdot\left(p\left(s_{n}\right)\right)=p(\infty)$, it develops that, for $a \notin B$,

$$
\begin{aligned}
p_{2}(a)= & E_{a}\left[\sum_{l \leqq n \wedge n_{B}}\left(p_{2}\left(s_{l-1}\right)-p_{2}\left(s_{l}\right)\right)+p_{2}\left(s_{n \wedge n_{B}}\right)\right] \\
= & E_{a}\left[\sum_{l \leqq n n_{B}} e_{2}\left(s_{l-1}\right)\right]+E_{a}\left(p_{2}\left(s_{n_{B}}\right), n \geqq n_{B}\right) \\
& \quad+E_{a}\left(p_{2}\left(s_{n}\right), n<n_{B}\right) \\
\rightarrow & E_{a}\left[\sum_{l \leqq n_{B}} e_{2}\left(s_{l-1}\right)\right]+ \\
\quad & E_{a}\left(p_{2}\left(s_{n_{B}}\right), n_{B}<+\infty\right) \\
& \quad+p_{2}(\infty) P_{a}\left(n_{B}=+\infty\right) \quad(n \uparrow+\infty) \\
\geqq & E_{a}\left[\sum_{l \leqq n_{B}} e_{1}\left(s_{l-1}\right)\right]+E_{a}\left(p_{1}\left(s_{n_{B}}\right), n_{B}<+\infty\right) \\
= & +p_{1}(\infty) P_{a}\left(n_{B}=+\infty\right) .
\end{aligned}
$$

We learn from 3.15 that $p_{B}$ is the greatest excessive $p=\mathbf{K} e+p(\infty)$ with $e=0$ off $B, p \leqq 1$ on $B$, and $p(\infty) \leqq P$.(B).

Also (and this will be useful for us in Section 4), $p_{B}=1$ on $B$, so that, if, for two potentials $p_{1}$ and $p_{2}, p_{2} \geqq p_{1}$ on $B$ and $e_{1}, e_{2}=0$ off $B$, then, writing $e p$ for $\sum_{b \in B} e(b) p(b)$,

$$
e_{2}(B)=e_{2} p_{B}=e_{B} p_{2} \geqq e_{B} p_{1}=e_{1} p_{B}=e_{1}(B) ;
$$

in short, $e_{2}(B) \geqq e_{1}(B)$, a fact due to Gauss [1, pp. 37-39] for the case of Newtonian potentials.

\section{Capacities}

Given a set $B$ of lattice points for which $P .(\mathbf{B})=0$, its capacity $C(B)$ is the total charge

$$
C(B)=e_{B}(B)=\sum_{a \epsilon \partial B} P_{a}\left(n_{B}\left(w_{1}^{+}\right)=+\infty\right)
$$

of the electrostatic distribution $e_{B}$.

When $|B|(=$ the number of points of $B)=+\infty, C(B)=+\infty$; for, if $C(B)<+\infty$, then (use 2.7) $p_{B}$ converges to 0 at $\infty$, and, since $p_{B}=1$ on $B$, $|B|<+\infty$.

We shall therefore confine our attention to the capacities of finite sets $B$.

The following rules are helpful for computing $C(B)$ :

$$
\begin{aligned}
& C(B)=C(\partial B), \\
& C\left(B_{1}\right)=C\left(B_{2}\right),
\end{aligned}
$$$$
B_{1} \equiv B_{2}
$$

$$
C(B)=\max e(B): \quad e \geqq 0, \quad e=0 \text { off } B, \quad p=\mathbf{K} e \leqq 1,
$$$$
C\left(B_{1} \cap B_{2}\right)+C\left(B_{1} \cup B_{2}\right) \leqq C\left(B_{1}\right)+C\left(B_{2}\right) .
$$ 
4.2 is clear. $B_{1} \equiv B_{2}$ means that $B_{1}$ is congruent to $B_{2}$ (with respect to orthogonal transformations with integral entries). 4.3 is then clear. $p=\sum_{b \in B} K(a, b) e,(b) \leqq 1$ implies $p_{B}-p \geqq 0$, and using 3.16 to compute the (nonnegative) total charge $C(B)-e(B)$ of $e_{B}-e$ proves 4.4 .4 .5 gets a similar proof: the inclusion

$$
\left(w: n_{B_{1} \cup_{B_{2}}}<+\infty, n_{B_{2}}=+\infty\right) \subset\left(w: n_{B_{1}}<+\infty, n_{B_{1} \cap^{B_{2}}}=+\infty\right)
$$

implies $p_{B_{1} \mathrm{U}_{B_{2}}}-p_{B_{2}} \leqq p_{B_{1}}-p_{B_{1} \cap^{B_{2}}}$; now compute the (nonnegative) total charge of $e_{B_{1}}+e_{B_{2}}-e_{B_{1} \cup_{B_{2}}}-e_{B_{1} \cap^{B_{2}}}$.

Given $B, B_{1}, B_{2}, \cdots, B_{n}$, let us write $\mathfrak{m}$ for subsets of $1,2, \cdots, n$, $|\mathrm{m}|=l$ for the number of points in $\mathrm{m}$, and $B_{\mathfrak{m}}=\bigcup_{i \in \mathfrak{m}} B_{i}$; it is clear from ${ }^{10}$

$$
P .\left(\cap_{l \leqq n} C\right)=-\sum_{l \leqq n}(-)^{l} \sum_{|\mathrm{m}|=l} P .\left(C_{\mathrm{m}}\right)
$$

that

$$
\begin{aligned}
0 & \leqq P .\left(n_{B}=+\infty, n_{B \cup B_{l}}<+\infty, l \leqq n\right) \\
& =-P .\left(n_{B}<+\infty\right)+P .\left(n_{B \cup B_{l}}<+\infty, l \leqq n\right) \\
& =-P .\left(n_{B}<+\infty\right)-\sum_{l \leqq n}(-)^{l} \sum_{|\mathfrak{m}|=l} P .\left(n_{B \cup B_{\mathfrak{m}}}<+\infty\right) \\
& =p_{B}-\sum_{l \leqq n}(-)^{l} \sum_{|\mathfrak{m}|=l} p_{B \cup B_{\mathfrak{m}}},
\end{aligned}
$$

and by using 3.16 to compute the (nonnegative) total charge of $-e_{B}-\sum_{l \leqq n}(-)^{l} \sum_{|\mathfrak{m}|=l} e_{B \mathbf{U}_{B_{\mathfrak{m}}}}$, it results that

$$
C(B)+\sum_{l \leqq n}(-)^{l} \sum_{|\mathfrak{m}|=l} C\left(B \cup B_{\mathfrak{m}}\right) \leqq 0 .
$$

G. Choquet [1, pp. 147-153] proved the counterpart of 4.8 for Newtonian potentials. 4.7 imitates G. Hunt [1, p. 53]. 4.5 is a special case of 4.8 $\left(n=2, B=B_{1} \cap B_{2}\right)$.

The following technique for estimating $C(B)$ is useful for Section 6 . Given $B$, if $A$ is the sum of $n(=|B|)$ solid cubes $[0,1]^{s}$ centered at the points of $B$, and if $\hat{C}(A)$ is the Newtonian capacity:

$$
\hat{C}(A)=\max \hat{e}(A): \hat{e} \geqq 0, \hat{e}=0 \text { off } A, \hat{p}(\xi)=\int_{A}|\xi-\eta|^{2-8} \hat{e}(d \eta) \leqq 1,
$$
then

$$
k_{4} \hat{C}(A) \leqq C(B) \leqq k_{5} \hat{C}(A),
$$

with $k_{4}, k_{5}$ depending on the dimension number $s$, but not on $B$.

To prove the overestimate, choose $k_{5}$ such that, for $\xi$ in the cube centered at $a$, the integral $\int|\xi-\eta|^{2-s} d \eta$ extended over the cube centered at $b$ is $\leqq k_{5} K(a, b)$, let $\hat{e}(d \eta)=e_{B}(b) d \eta$ on the cube centered at $b$, and estimate $\hat{p}(\xi)=k_{5}^{-1} \int_{A}|\xi-\eta|^{2-s} e(d \eta)$ in terms of $p_{B}$; the result is $\hat{p} \leqq 1$, and we conclude that

$$
4.11 \quad C(B)=e_{B}(B)=\hat{e}(A) \leqq k_{5} \hat{C}(A) .
$$

\footnotetext{
104.6 is dual to the classical inclusion and exclusion formula.
} 
To prove the underestimate, choose $k_{4}$ such that, for $\xi$ in the cube centered at $a$ and $\eta$ in the cube centered at $b, k_{4} K(a, b) \leqq|\xi-\eta|^{2-s}$, let $e(b)$ be the charge that the Newtonian electrostatic distribution $\hat{e}$ places on the cube centered at $b$, and estimate $p=k_{4} \sum_{b \in B} K(a, b) e(b)$ in terms of

$$
\hat{p}=\int|\xi-\eta|^{2-s} \hat{e}(d \eta) ;
$$

the result is $p \leqq 1$, and we conclude that

$$
C(B) \geqq k_{4} e(B)=k_{4} \hat{e}(A)=k_{4} \hat{C}(A) .
$$

Given compact $A \subset R^{s}$,

$$
\hat{C}(\alpha A)=\alpha^{s-2} \hat{C}(A),
$$

where $\alpha A$ is the set of points $\alpha x$ with $x \in A$.

We will use 4.13 for getting underestimates of $C(B)$; for example, if $B_{n}$ is the disc

$$
\left(l_{1}, l_{2}, \cdots, l_{s}\right):\left(l_{1}^{2}+l_{2}^{2}\right)^{1 / 2} \leqq n, \quad l_{3}=l_{4}=\cdots=l_{s}=0,
$$

then $C\left(B_{n}\right) \geqq k_{6} n^{s-2}$ for $n \uparrow+\infty$.

\section{Wiener's test}

Given a set $Q$ of lattice points, clustering to $\infty$, let $Q_{l}$ denote the intersection of $Q$ and the spherical shell $2^{l} \leqq|a|<2^{l+1}$, and let us prove Wiener's test: $5.1 P .(\mathrm{Q})=1$ or 0 according as $\sum_{l \geqq 1} 2^{-l(s-2)} C\left(Q_{l}\right)=$ or $<+\infty$.

When

$$
\begin{gathered}
\sum_{l \geqq 1} 2^{-l(s-2)} C\left(Q_{l}\right)<+\infty, \\
p_{Q_{l}}(a)=\sum_{b \in Q_{l}} K(a, b) e_{Q_{l}}(b) \leqq \sum_{b \in Q_{l}} k_{7}|a-b|^{2-s} e_{Q_{l}}(b) \\
\leqq 2 k_{7} 2^{-l(s-2)} C\left(Q_{l}\right),
\end{gathered}
$$

is the general term of a convergent sum, and an application of the first BorelCantelli lemma implies

$$
P .\left(n_{Q_{l}}=+\infty, l \uparrow+\infty\right)=1 ;
$$

2.6 implies $P .\left(\bigcup_{l \leqq n} \mathrm{Q}_{l}\right)=0$ for each $n \geqq 1$; and we infer that

$$
P .(\mathbf{Q})=0 \text {. }
$$

When

5.6

$$
\sum_{l \geqq 1} 2^{-l(s-2)} C\left(Q_{l}\right)=+\infty,
$$

$\sum_{l \geqq 1} 2^{-(4 l+k)(s-2)} C\left(Q_{4 l+k}\right)=+\infty$ for $k=0,1,2$, or 3 , and if we suppose, as we can, that $\sum_{l \geqq 1} 2^{-(4 l+1)(s-2)} C\left(Q_{4 l+1}\right)=+\infty$, it is clear that, if $m_{l}$ is the 
crossing time $^{11} \min \left(n: 2^{l} \leqq\left|s_{n}\right|<2^{l}+1\right)$, and if, for the moment, $s_{l}$ stands for the crossing place $s_{m_{l}}$, then for $l \uparrow+\infty$,

$$
\begin{aligned}
P_{s_{4 l}}\left(n_{Q_{4 l}+1}<m_{4 l+4}\right) & \geqq p_{Q_{4 l+1}}\left(s_{4 l}\right)-E_{s_{4 l}}\left[p_{Q_{4 l+1}}\left(s_{4 l+4}\right)\right] \\
= & \sum_{b \epsilon Q_{4 l} l+1}\left(K\left(s_{4 l}, b\right)-E_{s_{4 l}}\left[K\left(s_{4 l+4}, b\right)\right]\right) e_{Q_{4 l+1}}(b) \\
\geqq & k_{8}\left[\frac{2}{3}\left(2^{4 l+2}-2^{4 l}\right)^{2-s}-\frac{3}{2}\left(2^{4 l+4}-2^{4 l+2}\right)^{2-s}\right] C\left(Q_{4 l+1}\right) \\
\geqq & k_{9} 2^{-(4 l+1)(s-2)} C\left(Q_{4 l+1}\right)=t_{l},
\end{aligned}
$$

which implies

$$
\begin{aligned}
& P_{s_{4 l}}\left(n_{Q}=+\infty\right) \leqq E_{s_{4 l}}\left[n_{Q_{4 l+1}} \geqq m_{4 l+4}, P_{s_{4 l+4}}\left(n_{Q}=+\infty\right)\right] \\
& \leqq E_{s_{4} l}\left[n_{Q_{4 l+1}} \geqq m_{4 l+4}, E_{s_{4 l+4}}\left(n_{Q_{4 l+5}} \geqq m_{4 l+8}, P_{s_{4 l+8}}\left(n_{Q}=+\infty\right)\right)\right],
\end{aligned}
$$

5.8

$$
\begin{aligned}
& \leqq\left(1-t_{l}\right)\left(1-t_{l+1}\right)\left(1-t_{l+2}\right) \cdots \\
& =0
\end{aligned}
$$

and we conclude that

$$
P .\left(n_{Q}\left(w_{m_{4 l}}^{+}\right)<+\infty\right)=E \cdot\left(P_{s_{4 l}}\left(n_{Q}<+\infty\right)\right)=1, \quad l \uparrow+\infty .
$$

which completes the proof of 5.1.

\section{Thorns}

Given nonnegative $i(1) \leqq i(2) \leqq \cdots$, let $Q$ denote the thorn

$$
\left(l_{1}, l_{2}, \cdots, l_{s}\right):\left(l_{1}^{2}+l_{2}^{2}+\cdots+l_{s-1}^{2}\right)^{1 / 2} \leqq i\left(l_{s}\right), \quad l_{s} \geqq 1 .
$$

We use Wiener's test to prove that for $s \geqq 4$ dimensions

$$
6.1 P .(\mathrm{Q})=1 \text { or } 0 \text { according as } \sum_{n \geqq 1}\left(2^{-n} i\left(2^{n}\right)\right)^{s-3}=\text { or }<+\infty ;^{12}
$$

as an example, if $l \geqq 1$, and if $i(n)=n\left(\lg n \lg _{2} n \cdots\left(\lg _{k} n\right)^{\alpha}\right)^{-1 / s-3}{ }^{13}$ then $P .(\mathrm{Q})=1(0)$ for $\alpha \leqq 1(>1)$.

When $\lim \sup _{n \uparrow+\infty} n^{-1} i(n)>\alpha>0,2^{-n} i\left(2^{n}\right)>\alpha / 2$ for an infinite number of integers $n$; for such $n, Q_{n}$ contains the set $Q_{n}^{-}$of lattice points of a sphere of diameter $\geqq \min (1, \alpha) 2^{n} ; C\left(Q_{n}\right) \geqq C\left(Q_{n}^{-}\right) ; C\left(Q_{n}\right)$ is then $\geqq k_{10} 2^{n(s-2)} ;$ and the upshot is

$$
+\infty=\sum_{n \geqq 1}\left(2^{-n} i\left(2^{n}\right)\right)^{s-3}=\sum_{n \geqq 1} 2^{-n(s-2)} C\left(Q_{n}\right),
$$

which checks with 6.1 .

${ }^{11} m_{l}<+\infty$ for paths crossing from $|a| \leqq 2^{l}$ to $|a|>2^{l}+1$; for if

$$
\left(l_{1}^{2}+l_{2}^{2}+\cdots+l_{s}^{2}\right)^{1 / 2} \leqq 2^{l},
$$

then $\left(l_{1} \pm 1\right)^{2}+l_{2}^{2}+\cdots+l_{s}^{2}=l_{1}^{2}+l_{2}^{2}+\cdots+l_{s}^{2} \pm 2 l_{1}+1 \leqq 2^{2 l}+2 \cdot 2^{l}+1=$ $\left(2^{l}+1\right)^{2}$.

${ }^{12} 6.1$ is to be compared with Lebesgue's thorn: see Courant and Hilbert [1, pp. 272274].

${ }^{13} \lg _{1}=\lg$ and $\lg n=\lg \left(\lg _{n-1}\right)$ for $n \geqq 2$. 
When

$$
\lim _{n \uparrow+\infty} n^{-1} i(n)=0,
$$

$Q_{n}$ is long and thin, a sphere is not a good approximation, and we consider instead the ellipsoids $Q_{n}^{-} \subset Q$ and $Q_{n}^{+} \supset Q$ :

$$
\begin{aligned}
& Q_{n}^{-}=\left(l_{1}, l_{2}, \cdots, l_{s}\right): \frac{l_{1}^{2}+l_{2}^{2}+\cdots+l_{s-1}^{2}+\frac{\left(l_{s}-3 \cdot 2^{-n-1}\right)^{2}}{2^{2(n-1)}} \leqq 1,}{i\left(2^{n}\right)^{2}} \\
& Q_{n}^{+}=\left(l_{1}, l_{2}, \cdots, l_{s}\right): \frac{l_{1}^{2}+l_{2}^{2}+\cdots+l_{s-1}^{2}+\frac{\left(l_{s}-3 \cdot 2^{n-1}\right)^{2}}{2 \cdot i\left(2^{n+1}\right)^{2}} \leqq 1}{2 \cdot 2^{2(n-1)}} \leqq 1
\end{aligned}
$$

and compare the capacities $C\left(Q_{n}^{-}\right), C\left(Q_{n}^{+}\right)$to the Newtonian capacity of the solid ellipsoid

$$
E=\left(x_{1}, x_{2}, \cdots, x_{s}\right): \frac{x_{1}^{2}}{e_{1}^{2}}+\frac{x_{2}^{2}}{e_{2}^{2}}+\cdots+\frac{x_{s}^{2}}{e_{s}^{2}} \leqq 1, \quad e_{1}, e_{2}, \cdots, e_{s}>0 .
$$

The Newtonian capacity of $E$ is known; up to a factor depending on the dimension number, it is the reciprocal of the elliptic integral

$$
\mathbf{e}=\int_{0}^{+\infty} \frac{d t}{\sqrt{ }\left(e_{1}^{2}+t\right)\left(e_{2}^{2}+t\right) \cdots\left(e_{s}^{2}+t\right)} .
$$

The reader will find a neat proof of 6.4 for $s=3$ in G. Chrystal [1, p. 30].

When $\alpha=e_{1} / e_{s}=e_{2} / e_{s}=\cdots=e_{s-1} / e_{s}$,

$$
\begin{aligned}
\mathbf{e} & =\int_{0}^{+\infty}\left(\alpha^{2} e_{s}^{2}+t\right)^{-(s-1) / 2}\left(e_{s}^{2}+t\right)^{-1 / 2} d t \\
& =e_{s}^{2-s} \int_{0}^{+\infty}\left(\alpha^{2}+t\right)^{-(s-1) / 2}(1+t)^{-1 / 2} d t \\
& \sim e_{s}^{2-s} \int_{\alpha^{2}}^{1} t^{-(s-1) / 2} d t \\
& \sim e_{s}^{2-s} \frac{1}{2}(s-3) \alpha^{-(s-3)}
\end{aligned}
$$

by using 4.10 , it is plain that

$$
\begin{aligned}
& k_{11} 2^{+n(s-2)}\left(2^{-n} i\left(2^{n}\right)\right)^{s-3}<C\left(Q_{n}^{-}\right) \\
& \quad \leqq C(Q) \leqq C\left(Q_{n}^{+}\right)<k_{12} 2^{+n(s-2)}\left(2^{-(n+1)} i\left(2^{n+1}\right)\right)^{s-3}, \quad n \uparrow+\infty ;
\end{aligned}
$$

and an application of Wiener's test completes the proof of 6.1 .

When $s=3, P$.(Q) $=1$ even for the thinnest thorn $Q=\bigcup_{n \geqq 1}(0,0, n)$; $C\left(Q_{n}\right)$ is then $>k_{13} 2^{n}$, and Wiener's sum is $+\infty$.

We consider, instead, the set $Q=\bigcup_{n \geqq 1}(0,0, i(n))$ with integral $i(1)<i(2)<\cdots$ and prove that if

$$
i(n)-i(n-1) \geqq \lg i(n-1), \quad n \uparrow+\infty,
$$


then

6.8

$$
P .(\mathbf{Q})=1 \text { or } 0 \text { according as } \sum_{n \geqq 1} i(n)^{-1}=\text { or }<+\infty \text {; }
$$

as an example, if $i(n)=\left[n \lg n \lg _{2} n \cdots\left(\lg _{k} n\right)^{\alpha}\right],{ }^{14}$ then $P .(\mathbf{Q})=1(0)$ for $\alpha \leqq 1(>1)$.

Granting

$$
k_{14}\left|Q_{n}\right| \leqq C\left(Q_{n}\right) \leqq k_{15}\left|Q_{n}\right|
$$$$
n \uparrow+\infty
$$

it is clear that

$$
\begin{aligned}
& k_{14} \sum_{2^{n} \leqq i(l)<2^{n+1}} i(l)^{-1} \leqq k_{14} 2^{-n}\left|Q_{n}\right| \\
& \leqq 2^{-n} C\left(Q_{n}\right) \leqq k_{15} 2^{-n}\left|Q_{n}\right| \leqq 2 k_{15} \sum_{2^{n} \leqq i(l)<2^{n+1}} i(l)^{-1},
\end{aligned}
$$

and an application of Wiener's test proves 6.8. $C\left(Q_{n}\right) \leqq k_{15}\left|Q_{n}\right|$ with $k_{15}=K(0,0)^{-1}$ is immediate from 4.5 , and to complete the proof, it is enough to use 6.7 and 2.7 to check the estimate

$$
\begin{aligned}
\sum_{b \epsilon Q_{n}} K(a, b) \leqq & k_{16} \sum_{1 \leqq l \leqq\left|Q_{n}\right|}(\ln \lg 2)^{-1} \\
& <k_{17} n^{-1} \lg \left|Q_{n}\right| \leqq k_{17} \lg 2, \quad a \in Q_{n}, \quad n \uparrow+\infty,
\end{aligned}
$$

and to infer, from 4.4, that

$$
6.12 \quad C\left(Q_{n}\right) \geqq k_{14}\left|Q_{n}\right|, \quad k_{14}=\left(k_{17} \lg 2\right)^{-1}, \quad n \uparrow+\infty .
$$

Problem. When $i(n): n \geqq 1$ is the set of prime numbers, is $P$.(Q) $=1$ ? Were Gauss's law $n / \lg n$ for the number of primes $\leqq n$ exact, we could assert that $+\infty>k_{18} \geqq \sum_{b \epsilon Q_{n}} K(a, b)$ for $a \epsilon Q_{n}$ and conclude, as in 6.12 , that $C\left(Q_{n}\right) \geqq k_{18}^{-1}\left|Q_{n}\right| \geqq k_{19} n^{-1} 2^{n}$ and that $\sum_{n \geqq 1} 2^{-n} C\left(Q_{n}\right)=+\infty$.

\section{RefERENCES}

P. Bateman et AL.

1. Seminar on convex sets, The Institute for Advanced Study, Princeton, 1949-1950.

J. Capoulade

1. Sur quelques propriétés des fonctions harmoniques et des fonctions préharmoniques,

G. Choquet Mathematica (Cluj), vol. 6 (1932), pp. 146-151.

1. Theory of capacities, Ann. Inst. Fourier, Grenoble, vol. 5 (1953-1954), pp. 131-295.

G. Chrystal

1. Electricity, Encyclopaedia Britannica, $9^{\text {th }}$ ed. (1879), vol. 8, pp. 3-104.

R. Courant and D. Hilbert

1. Methoden der mathematischen Physik, Bd. 2, Berlin, Springer, 1937.

J. L. Doob

1. Stochastic processes, New York, Wiley, 1953.

2. Semimartingales and subharmonic functions, Trans. Amer. Math. Soc., vol. 77 R. J. DuFfin (1954), pp. 86-121.

1. Discrete potential theory, Duke Math. J., vol. 20 (1953), pp. 233-251.

\footnotetext{
${ }^{14}[\gamma]$ is the greatest integer $\leqq \gamma$.
} 
A. ERdélyi et al. (Bateman Manuscript Project)

1. Higher transcendental functions, Vol. I, New York, McGraw-Hill, 1953.

2. Tables of integral transforms, Vol. I, New York, McGraw-Hill, 1954.

K. F. Gauss

1. Allgemeine Lehrsätze in Beziehung auf die im verkehrten Verhältnisse des Quadrats der Entfernung wirkenden Anziehungs- und Abstossungs-Kräfte, Ostwald's Klassiker der exakten Wissenschaften, Nr. 2, Leipzig, 1889.

E. Hewitt and L. J. Savage

1. Symmetric measures on Cartesian products, Trans. Amer. Math. Soc., vol. 80 (1955), pp. $470-501$.

G. A. HunT

1. Markoff processes and potentials I, Illinois J. Math., vol. 1 (1957), pp. 44-93.

2. Markoff processes and potentials II, Illinois J. Math., vol. 1 (1957), pp. 316-369.

3. Markoff processes and potentials III, Illinois J. Math., vol. 2 (1958), pp. 151-213.

B. H. MURDoch

1. Preharmonic functions, Thesis, Princeton, 1952.

G. Póluya

1. Über eine A ufgabe der Wahrscheinlichkeitsrechnung betreffend die Irrfahrt in Strassen-

S. VeRBLUNSKY netz, Math. Ann., vol. 84 (1921), pp. 149-160.

1. Sur les fonctions préharmoniques (Deuxième note), Bull. Sci. Math. (2), vol. 74 (1950), pp. 153-160.

\section{Kyôto UnIVERSITY \\ KYôTO, JAPAN}

